\title{
A Review on the Traditional Methods and Novel Techniques for Detection of Formaldehyde Concentration in Fish
}

\author{
Deepika S. ${ }^{1 *}$, Robin David ${ }^{2}$, Shukriya Salim ${ }^{3}$, Swathi J. ${ }^{4}$, Diya Mariam Varghese ${ }^{5}$, Asmin Azad ${ }^{6}$ \\ ${ }^{1,3,4,5,6}$ M. Tech Student, APJ Abdul Kalam Technological University, Department of ECE, TKM \\ College of Engineering, Kollam, Kerala, India. \\ ${ }^{2}$ Research Scholar, APJ Abdul Kalam Technological University, Department of Mechanical \\ Engineering, TKM CE, Kollam, Kerala, India.
}

*Corresponding Author, Email: deeps.deepika89@gmail.com

\begin{abstract}
Fish is an inevitable part of Kerala's food style and around 3\% of the State's economy is attributed to Fisheries. The recent news that hit the headlines of Kerala was the seizing of formalin laced fish from many parts of Kerala. There are numerous conventional chemical methods for detecting formalin concentration in fish. The main drawback is that the majority of these techniques are time-consuming, tedious and destructive that requires welltrained operators and cannot be applied for on-field detection. In this current paper, the hazardous effects of formalin in human body, traditional methods used at present and the recently developed novel techniques for effective detection of formaldehyde concentration in fish, are analyzed. An overview of the conventional methods- chemical methods and microbial attributes are discussed. The novel techniques include high performance liquid chromatography (HPLC), electrochemical biosensors, catalytic kinetic flurimetry technique, fluorescence spectroscopy, photonic crystal fiber based sensor, enhanced Raman spectroscopy (SERS) technique and optical fiber bundle sensor. Also, the advantages of these techniques over traditional methods are pointed out. The novel techniques exhibit good linearity, stability, sensitivity, non-invasive, non-destructive and most of them have the potential to be developed as a portable device. Using this non-destructive technique, unethical means of food preservation can be monitored, thereby ensuring fish quality in Kerala. Even though there has been great advancement in this field, traditional methods are still dominant in practical applications. It is high time that new techniques find their way out, because the quality of fish consumed by the people of this State is decreasing by a quick rate. Therefore, further efforts are necessary to facilitate early adoption of novel technologies in the fish industry and promote their standardization to complement or replace current legislation standards for the evaluation of fish quality.
\end{abstract}

Keywords - HPLC, electrochemical biosensor, SERS, optical fiber bundle sensor

\section{INTRODUCTION}

Formaldehyde is a noxious chemical extremely threatening to human life causing malicious diseases like cancer and leukemia. Due to the abundant endogenous enzymes and psychrophilic bacteria in fish bodies and the fragile organization of fish, fish and fish products are easily perishable and are affected by pre-harvest and postmortem factors, thus decreasing their value. If the point of sale is far away from the place of catch, formalin is used as the preservative and ammonia mixed with frozen water is used to keep the fish fresh [1]. Hence, it is significant to evaluate fish freshness in an effective and timely manner.

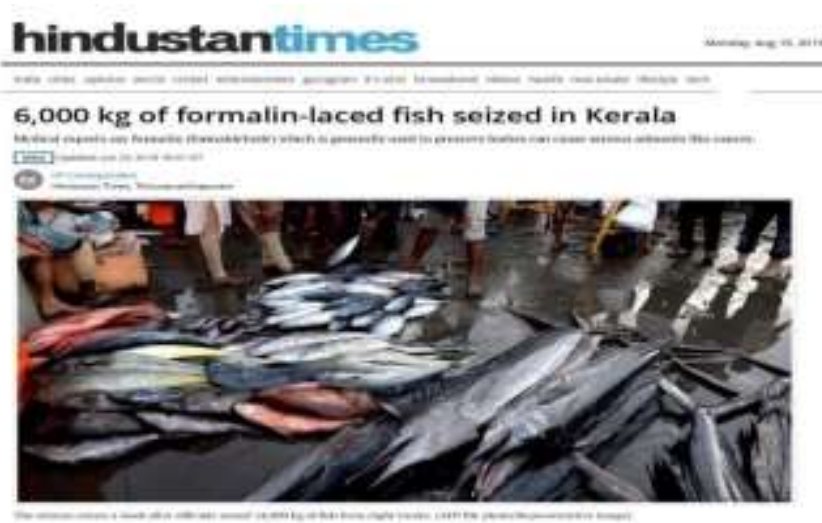

Fig. 1 Newspaper report regarding seizing of formalin laced fish in Kerala 
As a result of this highly sensitive issue, many techniques have been developed in the recent time for effective detection of formaldehyde concentration in fish. There are many conventional techniques available for evaluating the freshness of fish, including sensory evaluation techniques such as Quality Index Method (QIM), chemical detection techniques relying on instrumental measurements. Majority of these conventional techniques are tedious, timeconsuming and destructive, which require well trained operators and they cannot be applied on-field or for on-line detection [2-3].

With the rapid technological developments in electrochemistry, optics and mathematics, many novel nondestructive, rapid and cost-effective techniques have been developed for evaluating fish freshness, which can even achieve on-line detection. The current review is aimed to summarize and compare various kinds of novel technologies developed in the recent past [2]. These techniques include high performance liquid chromatography (HPLC), electrochemical biosensors, catalytic kinetic flurimetry technique, fluorescence spectroscopy, photonic crystal fiber based sensor, enhanced Raman spectroscopy (SERS) technique and optical fiber bundle sensor. In this review paper, the principles and applications of these techniques are summarized, their advantages and disadvantages are compared, and some challenges and future research trends are discussed.

\section{HAZARDOUS EFFECTS OF FORMALIN IN HUMAN BODY}

Inhaling or consumption of formalin in great quantities can have the following effects in the human body:

- Burning impact in the nose, eyes, throat, watery eyes, coughing, skin irritation, nausea, wheeze, bronchitis and pneumonia. These symptoms are caused when the formaldehyde present in inhaling air exceeds $0.1 \mathrm{ppm}$.

- Continuous ingestion of formalin can lead to cancer, as it releases toxins into the body.

- Increased risk of leukemia, particularly myeloid leukemia.

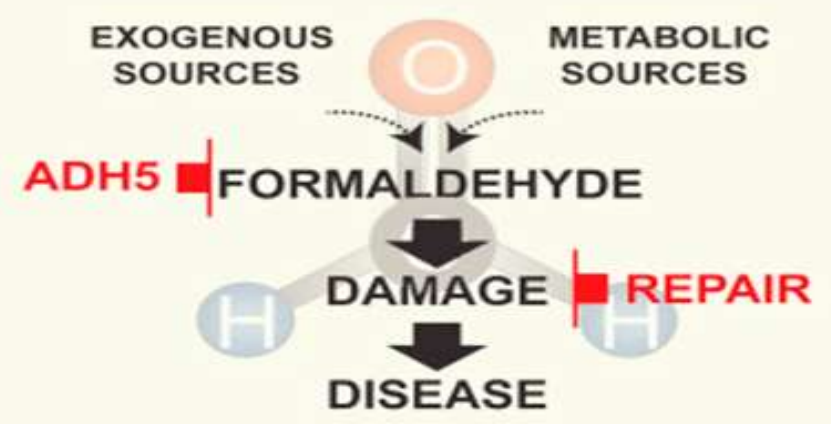

Fig.2: Effect of Formalin in Human Body
Formaldehyde is a poisonous chemical that can lead to sudden death, if swallowed. Formaldehyde/formalin produces gastritis in the stomach, that greatly varies in character. As a result, the inflammatory process may take place in the upper jejunum and duodenum [4]. In whichever form the chemical- formalin is instigated or absorbed in the body, it can lead to abrasions in the parenchymatous organs. Due to absorption of this chemical, severe or mild type of cloudy swelling appears in the liver, which is followed by change in the leukocyte infiltration, cell nuclei and vacuolation of protoplasm. Hence, it leads to focal necrosis. Also, due to inhalation of formalin, similar symptoms and diseases follow [4]. Many changes take place in liver and kidneys, such as, fat degeneration, leukocyte infiltration, focal necrosis and cloudy swelling. It also adversely affects the respiration channel and reproduction system [5].

\section{TRADITIONAL FORMALDEHYDE DETECTION METHODS}

Conventional chemical analysis comprises many steps. But, a majority of these are not understood by common people. It can be analyzed by a trained person only. The fish sample needs to be treated with chemical reagents and the chemical reactions need to be analyzed, which is quite challenging. It is required to have a proper understanding of the nature of reaction of the fish sample with chemicals. Moreover, the laboratory intended to carry out the experiment should hold appropriate equipment and facilities- reagents, solvents, glassware, testing apparatus, water, electricity, etc.

Conventional microbial methods, such as plate colonycounting methods are laborious and costly, and require complex sample preparation procedure and trained personnel. Therefore, rapid microbiological inspection techniques have been developed during the last decade. However, the detection procedures in these techniques are still slow or inconvenient, which are difficult to meet the requirements of the food industry.

It is quite evident that the traditional techniques for evaluating fish freshness always require specialized persons and numerous steps, and are time-consuming, laborious and invasive. While conventional techniques always refer to unilateral indicator, which cannot completely assess the fish freshness, routine surveillance often needs to combine several parameters to objectively evaluate the freshness of fish.

Therefore, traditional detection methods have various disadvantages; particularly, they are destructive and cannot be used for on-line detection of chemicals. In order to achieve rapid, non-invasive, accurate, and even on-line detection of fish quality, more and more novel technologies have been developed for fish freshness, including biosensors, spectroscopies, and optical sensors. 


\section{NOVEL TECHNIQUES FOR FORMALDEHYDE DETECTION IN FISH}

High-performance liquid chromatography (HPLC) is a method that is utilized in analytical chemistry which is used to identify, quantify and separate individual elements in a mixture. Pumps are used to pass the pressurized liquid solvent that consists of the sample mixture through a filled column consisting of a solid adsorbent material. HPLC is one of the most relevant analysis methods in evaluating quality of fish, that are rapid, simple and cost-saving technique. The content of formalin in nine readily available freshwater, marine water and a shrimp species that are marketed in the city of Dhaka were analyzed using the HPLC techniques. 22.90 and $6.90 \mu \mathrm{g} \mathrm{kg}^{-1}$ were the attained limit of quantification and limit of detection values respectively. Various levels of formaldehyde were detected in the analyzed samples of fish. The observed levels of formaldehyde content in the shrimp samples, marine and freshwater samples varied from $13.57 \pm 1.93 \mathrm{mgkg}^{-1}$, $10.8 \pm 1.72 \quad-\quad 39.68 \pm 7.87 \mathrm{mg} \mathrm{kg}^{-1}$ and $5.1 \pm 0.71-$ $12.26 \pm 1.67 \mathrm{mg} \mathrm{kg}^{-1}$ respectively. Eventually, a range of $5.1 \pm 0.71-39.68 \pm 7.87 \mathrm{mg} \mathrm{kg}^{-1}$ formaldehyde content was found in majority of the marketed fish [6].

In electrochemical biosensors, the electrode has good electro-catalytic ability towards the redox reaction of some compounds derived from fish deterioration or can be absorbed by these putrefactions, which influences the electronic characteristics of the electrode, realizing the analysis of fish freshness. A rather inexpensive and effective technique for the formaldehyde content monitoring is the electrochemical method as a result of its high rapidity, sensitivity, economy and reliability. Utilizing $\mathrm{MB}$ as the redox indicator, a novel electrochemical biosensor was developed based on FDH/AuNPs/[EMIM][Otf] /CHIT/GCE. AuNPs dispersed onto chitosan and ionic liquid were used to enhance the surface for the immobilization of FDH. Thus, the detection sensitivity of the chemical- formaldehyde was also enhanced. From the obtained results, it was quite evident that the AuNPs gave a nano-sized domain for the proximate interaction between the modified electrode and enzyme (FDH) that are highly crucial for the coherent electron transfer directly. The advantages of the developed formaldehyde biosensor over conventional methods was that it exhibited excellent stability and stability and sensitivity. The observed linear co efficiency $\left(\mathrm{r}^{2}\right)$ was found to be 0.9787 , with a detection limit of $0.1 \mathrm{ppm}$ and rate of recovery- $81.2 \%$ to $82.2 \%$. Hence the biosensor developed exhibits a rapid, simple, highly selective, highly sensitive, extreme detection range and a suitable technique for detection of formaldehyde in the research industry based on fish [7].

A novel technique has been developed using a catalytic kinetic fluorimetry technique for determining trace formaldehyde in food samples. This is primarily based on the fact that formaldehyde in phosphoric acid catalyzes pyrroline $\mathrm{Y}$ oxidation by sodium periodidate. When the excitation wavelength changed into the set at $546 \mathrm{~nm}$, the decreased fluorescence intensity $(\Delta \mathrm{F})$ at $563 \mathrm{~nm}$ was found to be proportional to the concentration of formaldehyde in the range from 0 to $0.30 \mu \mathrm{g} \mathrm{mL}^{-1}$. The detection limit and the mean recovery of formaldehyde were $2.0 \times 10^{-2} \mathrm{ng}$ $\mathrm{mL}^{-1}$ and $101.3 \%(n=6)$, respectively. The current method was applied in aqueous products to assess trace formaldehyde and the observed results were in good agreement with those obtained by distillationspectrophotometry [8].

Ravindran et.al.[9] studied the utilization of various techniques based on spectroscopy to spot out adulteration in food. He analyzed the disadvantages of the wide variety of laboratory methods available at present. He put forth the idea which was based on spectroscopy which ushered an accurate, non-destructive and speedy technique to analyze adulterated food materials.

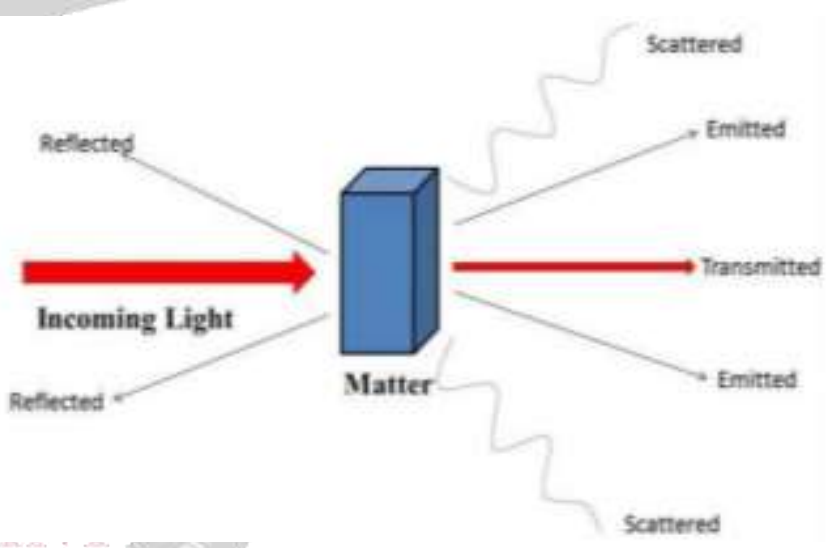

Fig. 3 Basic Principle of Spectroscopy

Various types of spectroscopy techniques were mentioned in this paper for the detection of adulteration. The methods include Fluorescence Spectroscopy, Ultraviolet and Visible Spectroscopy, NIR Spectroscopy, Fourier Transform Infrared Spectroscopy and Raman Spectroscopy.

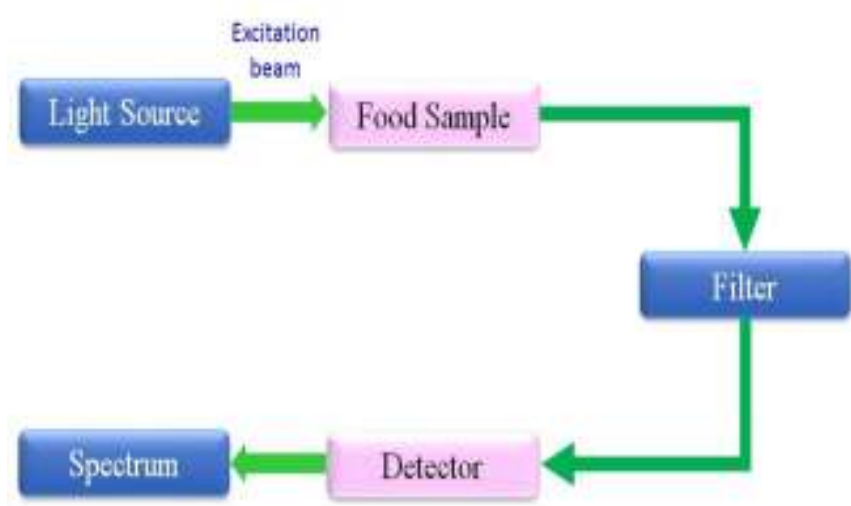

Fig.4: Block Diagram of Fluorescence Spectroscopy 
A simple, smart and reliable Photonic Crystal Fiber (PCF) based formaldehyde sensing method was introduced by Arif et.al. [10]. The optical characteristics like refractive index of formaldehyde was chosen for estimation. A comprehensive simulation was done based on the fact that a simple PCF pattern follows all the optical properties for the propagation of light. Thus, the proposed method employed a simple PCF structure which is handy and capable enough to detect the presence of formalin with a notable sensitivity.

Zhang et.al. [5] explained a simple on-site rapid measurement method for the detection of volatile formaldehyde in aquatic products using derivative reactionbased surface enhanced Raman spectroscopy (SERS) technology and a homemade portable purge-sampling device. Trace formaldehyde isolated from complex aquatic matrices was reacted with a derivative reagent via a purgesampling to generate a Raman active analyte for the consequent SERS analysis. $\mathrm{Au} / \mathrm{SiO}_{2}$ nanoparticles (NPs) have been used as the enhancement substratum to substantially increase in Raman signal intensity. The results indicate that the proposed technique is accurate and suitable for on-site applications.

Yet another innovative method to detect concentration of formaldehyde is the solid phase micro extraction (SPME)GC-MS method that is based on the fiber derivatization with pentafluorobenzyl-hydroxyl-amine hydrochloride [11]. $28 \mu \mathrm{g} \mathrm{kg}-1$ and $17 \mu \mathrm{g} \mathrm{kg}-1$ were the calculated limit of quantification and limit of detection values respectively. 12 different species of fish including crustaceans, freshwaterfish and sea-fish were assessed for determining the variable levels of formaldehyde in each species. Moreover, roasted, boiled, deep frozen, fresh fish and canned fish were also employed for the analysis. From the experimental results it was quite evident that samples of fish falling under the family of Gadidae was traced with the highest level of formaldehyde. The values ranged from $6.4 \pm 1.2 \mathrm{mg} \mathrm{kg}-1$ to $293 \pm 26 \mathrm{mg} \mathrm{kg}-1$. Less than $22 \mathrm{mg}$ $\mathrm{kg}-1$ level of formaldehyde was found in all other fish samples. Fish stored on ice was investigated and it showed moderate levels of formaldehyde at temperature around $0{ }^{\circ} \mathrm{C}$.

An efficient method for analyzing formalin concentration in frozen fish is the SPME-gas chromatography-ion trap tandem mass spectrometry technique [12]. For the experimental design, the consequences resulting from temperature of extraction, extraction time and stirring matrix were taken into consideration. The experimental environment that gave the optimum SPME extraction yield was found out by using the regression model calculated from the experimental design. Several factors such as precision, linearity, selectivity, trueness, limit of quantitation (LOQ) and limit of detection (LOD) were used for the validation procedure. LOQ values and LOD values were very low. On the other hand, linearity obtained was over one order of magnitude. Good precision was achieved both in terms of between-day precision and intra-day repeatability on two concentration levels (less than 9\%). The recovery values at $100 \mu \mathrm{g} \mathrm{kg}-1$ and $3 \mathrm{mg} \mathrm{kg}^{-1}$ were estimated as $82.3 \pm 1.8 \%$, and $95.3 \pm 1.2 \%(\mathrm{n}=3)$. The selectivity of the system has been demonstrated by the addition of 2, 3, 4, 5, 6-pentafluorobenzyl alcohol as an interfering substance. Finally, the applicability of the process to formaldehyde determination has been demonstrated in a variety of frozen fish samples.

The impact of formaldehyde (FA) on the value of the fluorescence ratio $\delta \mathrm{F}$ was studied by Aubourg [13]. A model system was established in which formaldehyde reacted with propylamine and fish muscle for 25 days at 30 ${ }^{\circ} \mathrm{C}$. It has been observed that formaldehyde has a lower ability to generate fluorescent compounds when compared to typical fish oxidation products, i.e. Propanal and hexanal. Nevertheless, in the presence of both lipid oxidation aldehydes, the formalin containing mixtures resulted in a higher value for $\delta \mathrm{F}$. It was observed that systems containing fatty fish (sardine) muscle and formaldehyde produced better fluorescence than lean fish (cod) and formaldehyde. This is due to formation of lipid oxidation compounds under the reaction conditions of the former systems. Thus, formalin content in a reacting medium increases the fluorescence formation, so that $\delta F$ could be used as an appropriate measure for damage of fish.

The optical fiber bundle-based sensor has been experimentally demonstrated by Yasin et.al. [1] to test the concentration of formaldehyde in fish. The intensity of the backscattered radiation was measured and analyzed to test formaldehyde concentration when contaminated fish samples were illuminated with a red laser light. As it is nondestructive, it has wide applications for developing alloptical inexpensive and portable sensors for the detection of toxic chemicals and pollutants in the food industry and agriculture sector and is suitable for measurements in this area.

Diverse efforts have been geared in recent years towards the production of optical fiber sensors. It provides many advantages over other sensor types such as remote sensing, immunity to electromagnetic and radio frequency interference, small size, and multiplexing information from a large number of sensors in a single cable. In this paper, the author attempts to put forth a simple yet effective formalin detection scheme, which is constructed based on gathering and evaluation of back reflected laser light from the food sample (here the food sample is fish, in particular). The experimental results based on the fiber optic sensor are expected to be of use for the onsite detection of formaldehyde concentration in fish [1].

A simple experimental setup was used to demonstrate the working of an optical fiber bundle sensor. The experimental 
setup consists of a Helium-Neon emitting red laser light with wavelength $633 \mathrm{~nm}$, a digital Voltmeter and a Newport SL-818 Optical Detector.

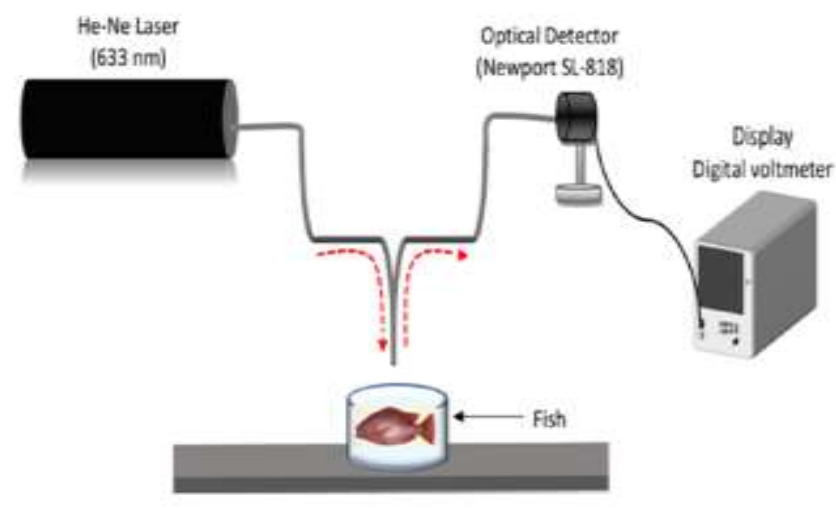

Fig. 5: Experimental Setup

The fish samples were illuminated by using a He-Ne laser via a two-port fiber bundle probe. The fiber bundle collects the intensity of light reflected from the fish through the second port of the fiber bundle probe. It was then measured by means of the optical detector, which is then related to a digital voltmeter. The fiber bundle probe was mounted on a support with micrometric motion, which enabled fine control of the distance between the sample and the prob. Fig.5 illustrates a schematic representation of the experimental setup.

The optical fiber bundle sensor was tested on two distinct types of common fish: the Gourami and the Snapper fish. Both types of fish were dipped in formalin for 60 minutes. The detector's output voltage was determined by varying the fiber bundle probe's distance from the head, from 0 to $1 \mathrm{~mm}$ in steps of $50 \mu \mathrm{m}$. The procedure is repeated for eight distinct formaldehyde concentrations in water ranging from $0 \%$ to $21 \%$ in increments of $3 \%$ (Eight separate fish samples were used for each species, one for each concentration of formaldehyde).

\section{RESULTS AND DISCUSSIONS}

The present work reports an up to date review of traditional and novel techniques for formaldehyde detection in fish. Compared with conventional evaluation techniques, the innovative techniques have been demonstrated to be nondestructive, non-invasive, and rapid methods, which can be applied in sorting and classification of fish based on formaldehyde concentration, or on-line. Moreover, these techniques are environmentally friendly and highly effective due to minimal or no chemical reagent and sample preparation. Among the methods, the spectroscopy based and optical fiber bundle based formaldehyde detectors are found to be more efficient due to their detection based on light scattering and reflection property, but are costly compared to all other techniques.

\section{CONCLUSION}

It is noted that in spite of developing new techniques, traditional methods are still dominant in practical applications. Therefore, further efforts are necessary to facilitate early adoption of novel technologies in the fish industry and promote their standardization to complement or replace current legislation standards for the evaluation of fish quality. Although there is still a long way to apply these novel techniques as routine industrial assessment tools for fish freshness, it is still worth making efforts and investments in these technologies due to their great potential in becoming standardized methods for determination of formaldehyde concentration in fish.

\section{REFERENCES}

[1] M.Yasin, N. Irawati, A H Zaidan, Kismiyati, A T Mukti, A Soegianto, D K P Rosalia, R A Wardani, M Khasanah, H J Kbashi, A M Perego," Fiber bundle sensor for detection of formaldehyde concentration in fish", Elsevier, 1068-5200/ @ 2019 Elsevier Inc.

[2] Leilei Wu, Hongbin Pu, Da-Wen Sun," Novel techniques for evaluating freshness quality attributes of fish: A review of recent developments",Elsevier, 0924-2244/ ( ) 2018 Elsevier Ltd

[3] Hernán Reingruber, Lucas Blas Pontel," Formaldehyde metabolism and its impact on human health, Current Opinion in Toxicology (2018)," Elsevier, volume 9, June 2018, Pages 28-34

[4] https://www.chromedia.org/chromedia?waxtrapp=mkqjtbEsHonOvm OIIEcCArB\&subNav=cczbdbEsHonOvmOlIEcCArBP

[5] Zhang Z, Zhao C, Ma Y, Li G,"Rapid analysis of trace volatile formaldehyde in aquatic products by derivatization reaction-based surface enhanced Raman spectroscopy", Analyst 139 (2014) 36143621

[6] Shuva Bhowmik, A. K. M. Nowsad Alam “ Determination of formaldehyde in wet marketed fish by HPLC analysis: A negligible concern for fish and food safety in Bangladesh", The Egyptian Journal of Aquatic Research, Vol. 43, Issue 3, September 2017, Pages 245-248

[7] Bohari Noor Aini, Shafiquzzaman Siddique and Kamaruzaman Ampon, Biosensors (Basel), Published on June 2016

[8] Guirong Li and Lijuan Han, "Determination of formaldehyde in aquatic products by a sensitive catalytic fluorescence method", Anal. Methods, 2014, 6, 426-432, issue 2

[9] Ajith Ravindran, Flavia Princess Nesamani, Nirmal D,"A Study on the use of Spectroscopic Techniques to Identify Food Adulteration", IEEE, 978-1-5386-0576-9/18/\$31.00 @2018 IEEE

[10] Md. Faizul Huq Arif, Md. Jaminul Haque Biddut, Kawsar Ahmed, Sayed Asaduzzaman," Simulation Based Analysis of Formalin Detection Through Photonic Crystal Fiber",IEEE, 978-1-5090-12695/16/@2016 IEEE

[11]F. Bianchi M. Careri M. Musci A. Mangia, 'Fish and food safety: Determination of formaldehyde in 12 fish species by SPME extraction and GC-MS analysis', Food Chemistry, Volume 100, Issue 3, 2007, Pages 1049-1053

[12]F. Bianchi, M. Careri, C. Corradini, M. Musci and A. Mangia, "Innovative Method for Ultratrace Determination of Formaldehyde in Frozen Fish: SPME Extraction and GC-ITMS/MS Analysis", Current Analytical Chemistry (2005) 1: 129. https://doi.org/10.2174/1573411054021600

[13] Aubourg, S. Influence of formaldehyde in the formation of fluorescence related to fish deterioration. Z Lebensm Unters Forsch 206, 29-32 (1998) https://doi.org/10.1007/s002170050208 